\title{
On Causality and Dynamic Stability of Perfectly Matched Layers for FDTD Simulations
}

\author{
F. L. Teixeira and W. C. Chew, Fellow, IEEE
}

\begin{abstract}
We investigate the spectral properties of the Cartesian, cylindrical, and spherical perfect matched layer (PML) absorbing boundary conditions. In the case of the anisotropicmedium PML formulation, we analyze the analytical properties of the constitutive PML tensors on the complex $\omega$-plane. In the case of the complex-space PML formulation (complex coordinate stretching formulation), we analyze the analytical properties of field solutions directly. We determine the conditions under which the PML's satisfy (or do not satisfy) causality requirements in the sense of the real-axis Fourier inversion contour. In the case of the noncausal PML, we point out the implications on the dynamic stability of time-domain equations and finite-difference time-domain (FDTD) simulations. The conclusions have impact both on the design of PML's for practical FDTD simulations and on the use of PML's as a physical basis for engineered artificial absorbers on nonplanar (concave or convex) surfaces. Numerical results illustrate the discussion.
\end{abstract}

Index Terms-Absorbing boundary conditions, anisotropic media, dispersive media, FDTD methods, perfectly matched layer.

\section{INTRODUCTION}

$\mathbf{T}$ WHE perfectly matched layer (PML) absorbing boundary condition (ABC), introduced by Berenger [1], [2] is a very efficient means to truncate the computational domain in the finite-difference time-domain (FDTD) simulation of electromagnetic (EM) problems [1]-[11]. Being a material $\mathrm{ABC}$, the PML retains the nearest neighbor interaction characteristic of the FDTD method, making it particularly attractive for parallel simulations. It has also been applied to finiteelement method (FEM) simulations [10], [12], [13], elasticwave problems [14], and paraxial-wave problems [15] with similar success.

More recently, the recognition that, via the complex coordinate stretching formulation [3], the PML can be interpreted as an analytic continuation of Maxwell's equations (ME's) to a complex variable spatial domain (or complex space, for short) [16], [17], [21], provided the basis for the extension of the PML to two-dimensional (2-D) cylindrical [20], [21], 3 -D cylindrical [22], and three-dimensional (3-D) spherical coordinates [20], [22] (extensions to 2-D cylindrical coordi-

Manuscript received March 25, 1998. This work was supported in part by the Air Force Office of Scientific Research under MURI Grant F49620-961-0025, by the Office of Naval Research under Grant N00014-95-1-0872, by the National Science Foundation under Grant NSF-ECS93-02145, and by a CAPES/Brasilia Graduate Fellowship.

The authors are with the Center for Computational Electromagnetics, Electromagnetics Laboratory, Department of Electrical and Computer Engineering, University of Illinois at Urbana-Champaign, Urbana, IL 61801-2991 USA.

Publisher Item Identifier S 0018-9480(99)04286-6. nates using different approaches are also considered in [18] and [19]).

One of the desirable properties of the artificial PML media in any of these coordinate systems is that it should retain the causality conditions observed by the original ME's. This naturally leads to the study of the analytic properties on the complex $\omega$-plane of either the PML tensor constitutive parameters (in case of anisotropic-medium PML's [6]-[9], [12], [13], [18], [22]), or the complex-space ME's Green's functions (in case of complex-space PML's [3], [5], [10], [14]-[17], [20], [21]).

In this paper, we study these analytic properties for the various coordinate system PML's: Cartesian, cylindrical, and spherical. The discussion is centered on the spectral properties of these PML's. Therefore, the conclusions do not depend on the peculiarities of the time-domain implementation (e.g., whether or not it utilizes field splitting), but have consequences on any time-domain implementation. In particular, we point out conditions under which causality is violated in the sense of a real-axis inverse Fourier contour and the consequences of this fact on the dynamic stability of the PML-FDTD simulations. The conclusions have impact on the design of PML's for FDTD simulations and on the use of PML's as a physical basis for engineered artificial absorbers on nonplanar (concave or convex) surfaces. Numerical results are presented to illustrate the discussion. Throughout this paper, the $e^{-i \omega t}$ convention will be used.

\section{Causality, Dynamic Stability, AND FOURIER INVERSION CONTOUR}

In this section, we examine the connection between the violation of causality in the sense of a real-axis Fourier inversion contour and the dynamic stability of the FDTD algorithm. The discussion is centered on the analytical properties of the $\overline{\boldsymbol{\epsilon}}(\omega)$ tensor. For brevity, we restrict our attention to the electric-field constitutive relation. By invoking the duality principle [23], all of the following conclusions also apply to the magnetic case.

In the frequency domain, the constitutive relation for the electric field is written as

$$
\boldsymbol{D}(\omega)=\bar{\epsilon}(\omega) \cdot \boldsymbol{E}(\omega)
$$

where $\bar{\epsilon}$ is the $3 \times 3$ permittivity tensor. In the time-domain, (1) becomes

$$
\boldsymbol{D}(t)=\int_{-\infty}^{\infty} \overline{\boldsymbol{\epsilon}}(\tau) \cdot \boldsymbol{E}(t-\tau) d \tau .
$$

Invoking causality, we have that $\overline{\boldsymbol{\epsilon}}(t)=0$ for $t<0$. 
By writing

$$
\overline{\boldsymbol{\epsilon}}(t)=\frac{1}{2 \pi} \int_{-\infty}^{\infty}[\overline{\boldsymbol{\epsilon}}(\omega)-\overline{\boldsymbol{\epsilon}}(\infty)] e^{-i \omega t} d \omega+\delta(t) \overline{\boldsymbol{\epsilon}}(\infty)
$$

where $\overline{\boldsymbol{\epsilon}}(\infty)$ is a real-valued constant tensor, it is possible to show [23] that, if the inversion contour of (3) is carried out along the real $\omega$-axis, the condition $\bar{\epsilon}(t)=0$ for $t<0$ implies that the integrand in (3) must be analytic (holomorphic) in the upper half-plane (zeros are also forbidden on the upper half-plane; for a more detailed discussion of the connection between causality and the analytical properties of the $\overline{\boldsymbol{\epsilon}}(\omega)$ tensor, see [24]-[26]). It should be noted here that when we refer to causality in the sense of a real-axis Fourier inversion contour, we are restricting ourselves to the primitive causality condition (i.e., the effect cannot precede the cause), as defined in [26]. The term causality also appears in other contexts. For instance, it is sometimes used to refer to the relativistic causality condition, which states that no signal can propagate with velocity greater than $c$ [26].

When $\overline{\boldsymbol{\epsilon}}(\omega)$ is not analytic in the upper half-plane, causality can still be preserved, provided the Fourier inversion contour is taken above any singularities [23]. In this case, the medium will behave as an active medium and its response will not be dynamically stable anymore. The definition of a dynamically stable system adopted here is such that all its eigenmodes approach zero as $t \rightarrow \infty$ (asymptotically stable) or remain bounded as $t \rightarrow \infty$ (marginally stable).

In FDTD simulations, the fields assume a causal behavior by the very nature of the method (explicit time-stepping scheme), regardless of the analytic properties on the frequency domain. This means that the Fourier inversion contour should always be taken above any singularities [23]. Therefore, following the discussion of the previous paragraph, violation of causality in the sense of real $\omega$-axis Fourier inversion contour implies a dynamically unstable FDTD method.

Note that the dynamic stability criterion is distinct from: 1) the numerical stability criterion that should be satisfied by a particular numerical discretization scheme. For a dynamically unstable system, no convergent numerical discretization schemes prevent the solutions from growing unbounded. For a further discussion on this aspect, see [27] (note, however, that the definition of dynamic stability in [27] is slightly different from the one adopted here and the anisotropic PML constitutive parameters considered there are frequency independent) and 2) dynamic stability is also a distinct criterion from the one incurred by a particular field-splitting of the modified ME's on the PML, which may induce a weak well posedness on the resultant system of equations, as discussed in [28]. Although the weak well posedness is also a characteristic of the system of partial differential equations in the continuum, we should stress that it is a distinct property from the dynamic stability issue discussed here. The discussion here is centered on the spectral characteristics of the PML equations and does not depend on the particular field-splitting scheme (if any) employed in the time-domain implementation of the PML.

When $\bar{\epsilon}(\omega)$ is analytic on the upper half $\omega$-plane, it can also be defined via the general time-domain relationship between
$\boldsymbol{E}(t)$ and $\boldsymbol{D}(t)$ as follows:

$$
\boldsymbol{D}(t)=\overline{\boldsymbol{\epsilon}}(\infty) \cdot \boldsymbol{E}(t)+\int_{0}^{\infty} \overline{\boldsymbol{\alpha}}(\tau) \cdot \boldsymbol{E}(t-\tau) d \tau
$$

[which is a particular case of (2)], through [24], [25]

$$
\overline{\boldsymbol{\epsilon}}(\omega)=\overline{\boldsymbol{\epsilon}}(\infty)+\int_{0}^{\infty} \overline{\boldsymbol{\alpha}}(\tau) e^{i \omega \tau} d \tau
$$

We immediately see that, if the function $\overline{\boldsymbol{\alpha}}(t)$ (generalized time-domain complex susceptibility kernel) is finite throughout the range of integration, the integral converges in the upper half-plane (real-axis excluded) and, therefore, $\bar{\epsilon}(\omega)$ is properly defined. In some instances, the integral in (5) diverges for $\omega$ on the real axis or in the lower half-plane, and definition (5) is invalid at these points. However, the function $\overline{\boldsymbol{\epsilon}}(\omega)$ can still be defined as the analytic continuation of (5) from the upper half-plane. It will, in general, contain singularities in these regions [24], [25]. This is the case, for instance, for an isotropic media with static conductivity, where the complex dielectric constant is usually written for real frequencies as the analytic continuation of (5) containing a pole at the origin

$$
\epsilon(\boldsymbol{r}, \omega)=\epsilon_{0}\left[\epsilon_{D}(\boldsymbol{r})+i \frac{\sigma(\boldsymbol{r})}{\omega \epsilon_{0}}\right] .
$$

The discussion in the last paragraph is directly related to the issue of causality of Cartesian PML's to be treated in Section III.

\section{CARTESIAN PML ANALYSIS}

In this section, we examine the causality and dynamic stability of the Cartesian PML.

\section{A. Anisotropic Formulation: Analytical Properties of the Constitutive Tensors}

Berenger first proposed the PML in Cartesian coordinates [1], [2] and, since then, Cartesian-grid FDTD codes have been implemented and tested in several radiation and scattering examples [1]-[11]. These examples use different anisotropic (unsplit) and split-field schemes where no instability have been observed. Since dynamic instability is usually a dramatic (early time) effect, we should, therefore, expect the Cartesian PML to be dynamically stable and causal in the sense of real-axis Fourier inversion contour. The analysis on this section will serve to verify this conjecture.

In the anisotropic-medium PML formulation [6]-[9], [12], the fields inside the PML region obey the ME's in an anisotropic medium with properly chosen constitutive tensors. Therefore, a useful test for violation of causality is to check if the resultant frequency-domain constitutive tensors $\overline{\boldsymbol{\epsilon}}(\boldsymbol{r}, \omega)=\bar{\epsilon} \overline{\boldsymbol{\Lambda}}(\boldsymbol{r}, \omega)$ and $\overline{\boldsymbol{\mu}}(\boldsymbol{r}, \omega)=\mu \bar{\Lambda}(\boldsymbol{r}, \omega)$ satisfy, along with the crossing relation $\overline{\boldsymbol{\Lambda}}^{*}(\boldsymbol{r}, \omega)=\overline{\boldsymbol{\Lambda}}\left(\boldsymbol{r},-\omega^{*}\right.$ ) (the star denotes complex conjugation), the Kramers-Kronig relations [23]-[25], [28]-[32]. This should also be true for their inverses $\overline{\boldsymbol{\epsilon}}(\boldsymbol{r}, \omega)^{-1}$ and $\overline{\boldsymbol{\mu}}(\boldsymbol{r}, \omega)^{-1}$ [24], [25].

The Cartesian PML constitutive tensor $\bar{\Lambda}(\boldsymbol{r}, \omega)$ is given by

$$
\overline{\boldsymbol{A}}(\boldsymbol{r}, \omega)=\hat{x} \hat{x}\left(\frac{s_{y} s_{z}}{s_{x}}\right)+\hat{y} \hat{y}\left(\frac{s_{z} s_{x}}{s_{y}}\right)+\hat{z} \hat{z}\left(\frac{s_{x} s_{y}}{s_{z}}\right)
$$


where, in usual time-domain simulations, the frequency dependence on the complex stretching variables $s_{\zeta}$ [3] $\zeta=x, y, z$ is given by the expression

$$
s_{\zeta}(\zeta, \omega)=a_{\zeta}(\zeta)+i \frac{\sigma_{\zeta}(\zeta)}{\omega}
$$

with $a_{\zeta}(\zeta) \geq 1$, and $\sigma_{\zeta}(\zeta) \geq 0$ in practical situations. The choice $\sigma_{\zeta}(\zeta)>0$ achieves the reflectionless absorption of propagating modes and the choice $a_{\zeta}(\zeta)>1$ achieves additional (faster) decay of evanescent modes (if they exist). Note that (8) resembles the expression of a complex dielectric constant of a conductive media.

Equation (7) is the most general form for an anisotropic Cartesian PML media and corresponds to a 3-D PML at a corner interface, where there is a simultaneous stretching on the three coordinates. This tensor is just the product of three simpler $3 \times 3$ uniaxial tensors

$$
\bar{\Lambda}(\boldsymbol{r}, \omega)=\bar{\Lambda}_{x}(x, \omega) \cdot \bar{\Lambda}_{y}(y, \omega) \cdot \bar{\Lambda}_{z}(z, \omega)
$$

with

$$
\bar{\Lambda}_{x}=\hat{x} \hat{x}\left(\frac{1}{s_{x}}\right)+(\hat{y} \hat{y}+\hat{z} \hat{z}) s_{x}
$$

and analogously for $\bar{\Lambda}_{y}(y, \omega)$ and $\bar{\Lambda}_{z}(z, \omega)$. In order for $\overline{\boldsymbol{\Lambda}}(\boldsymbol{r}, \omega)$ to satisfy causality, each of the $\overline{\boldsymbol{\Lambda}}_{\zeta}(\zeta, \omega)$ tensors must satisfy it individually (there are no pole-zero cancellations). Since the frequency dependence of $\bar{\Lambda}_{\zeta}(\zeta, \omega)$ and $\bar{\Lambda}_{\zeta}(\zeta, \omega)^{-1}$ is determined by $1 / s_{\zeta}$ and $s_{\zeta}$, the Kramers-Kronig relations must be satisfied individually by these functions. The Kramers-Kronig relations are a consequence of the application of the Cauchy's theorem to the function $\overline{\boldsymbol{\epsilon}}(\omega)-\overline{\boldsymbol{\epsilon}}(\infty)$ in the upper half-plane [23]. When presented in their usual form [23]-[26], [29]-[31], the analyticity of $\bar{\epsilon}(\omega)-\bar{\epsilon}(\infty)$ over the entire real axis is implicitly assumed. However, this is not the case, for instance, in conductive media, in which the complex dielectric constant has a term $i \sigma / \omega \epsilon_{0}$ and, therefore, has a pole singularity present at $\omega=0$. The Kramers-Kronig relations for conductive-like media must be properly modified to account for the deformation of the closed contour in the Cauchy's theorem to avoid the pole at the origin by means of an infinitesimal semicircle above it. This detail has been overlooked on a previous study on this topic [31]. In this more general form, the Kramers-Kronig relations read as [24], [25], [29]

$$
\begin{aligned}
\overline{\boldsymbol{\epsilon}}_{r}(\omega)-\overline{\boldsymbol{\epsilon}}(\infty) & =\frac{1}{\pi} P V \int_{-\infty}^{+\infty} \frac{\overline{\boldsymbol{\epsilon}}_{i}\left(\omega^{\prime}\right)}{\omega^{\prime}-\omega} d \omega^{\prime}, \\
\overline{\boldsymbol{\epsilon}}_{i}(\omega) & =-\frac{1}{\pi} P V \int_{-\infty}^{+\infty} \frac{\overline{\boldsymbol{\epsilon}}_{r}\left(\omega^{\prime}\right)-\overline{\boldsymbol{\epsilon}}(\infty)}{\omega^{\prime}-\omega} d \omega^{\prime}+\frac{\overline{\boldsymbol{\sigma}}}{\omega}
\end{aligned}
$$

where $\overline{\boldsymbol{\epsilon}}_{r}(\omega)$ and $\overline{\boldsymbol{\epsilon}}_{i}(\omega)$ are the real and imaginary parts of $\overline{\boldsymbol{\epsilon}}(\omega)$, $P V$ denotes the Cauchy principal value, and the integrals are carried out along the real axis. The $\overline{\boldsymbol{\epsilon}}_{r}(\omega)$ and $\overline{\boldsymbol{\epsilon}}_{i}(\omega)$ are to be interpreted as the limiting values as $\omega$ approaches the real axis from above. The last term in (12) is the pole contribution at the origin and represents the modification from the usual Kramers-Kronig relations. Whenever the stretching variables $s_{\zeta}$ are defined as having a pole at $\omega=0$, as in (8), the Kramers-Kronig relations to be used are as in (11)-(12). To apply relations (11) and (12) to (8), the following immediate identifications are made:

$$
\begin{aligned}
& \overline{\boldsymbol{\epsilon}}_{r}(\omega) \rightarrow a_{\zeta} \\
& \overline{\boldsymbol{\epsilon}}(\infty) \rightarrow a_{\zeta} \\
& \overline{\boldsymbol{\epsilon}}_{i}(\omega) \rightarrow \sigma_{\zeta} / \omega
\end{aligned}
$$

so that (11) and (12) become

$$
\begin{aligned}
0 & =\frac{1}{\pi} P V \int_{-\infty}^{+\infty} \frac{\sigma_{\zeta}}{\omega^{\prime}\left(\omega^{\prime}-\omega\right)} d \omega^{\prime} \\
\sigma_{\zeta} / \omega & =0+\sigma_{\zeta} / \omega .
\end{aligned}
$$

We immediately see that (14) verifies (12). Equation (13) also verifies (11) since [32]

$$
\frac{1}{\pi} P V \int_{-\infty}^{+\infty} \frac{\sigma_{\zeta}}{\omega^{\prime}\left(\omega^{\prime}-\omega\right)} d \omega^{\prime}=\pi \sigma_{\zeta} \delta(\omega)
$$

and, thus, (13) is true in the upper half-plane. As a result, (8) obeys the Kramers-Kronig relations, being causal in the sense previously stated. The fact that, in the real axis, we get a Dirac delta function from (15) is related to the divergence of the integral in (5) for real $\omega$. The expression (8) does not include the Dirac delta function since it must be treated as an analytic continuation from the upper half-plane expression in a similar fashion as in the (6). Such conclusions should be expected from the resemblance of (8) to the complex dielectric constant of a conductive medium. Indeed, given a general field relationship in the frequency domain

$$
\psi(\omega)=s_{\zeta}(\omega) \chi(\omega)=\left(a_{\zeta}+i \frac{\sigma_{\zeta}}{\omega}\right) \chi(\omega)
$$

this means to have, in the time-domain,

$$
\psi(t)=a_{\zeta} \chi(t)+\sigma_{\zeta} \int_{-\infty}^{t} \chi(\tau) d \tau
$$

where causality and (marginal) stability are evident.

A brief digression should be made at this point. In the case of the relationship $D(\omega)=(\epsilon+i \sigma / \omega) E(\omega)$, the marginal stability in (17) seems to contradict the fact that, for a conductive media, eigenfunctions with faster decay are expected. The point is that the marginal stability in this case is caused just by the definition of the $D(\omega)$ itself when incorporating conduction currents through the complex dielectric constant, as in (16). For instance, a DC conduction current $J(t)=$ $\sigma E(t)=\sigma E_{0} u(t)$ gives rise to linearly growing $D(t)$ field, and, similarly, an impulse current $J(t)=\sigma E(t)=\sigma E_{0} \delta(t)$ gives rise to a constant $D(t)$ field (which is an example of bounded eigenfunction that does not approach zero at $t \rightarrow \infty$ ). However, this marginal stability is not a property of the ME's themselves for conductive media since the term which appears in the equation for the $D(\omega)$ field (Ampere's law) is $i \omega D(\omega)$, and the pole at $\omega=0$ is cancelled. In fact, as $\sigma$ increases, the resultant eigenfunctions will have a faster decay since the factor $(-i \omega \epsilon+\sigma)^{-1}$ will have a pole that is shifted downwards in the lower half complex $\omega$-plane.

It is a simple exercise to verify that, if $a_{\zeta} \geq 1$ and $\sigma_{\zeta} \geq 0$, the function $1 / s_{\zeta}$ also satisfies the Kramers-Kronig relations. 
In this case, since there are no singularities present on the real axis, the usual form of Kramers-Kronig relations can be used.

In summary, the Cartesian anisotropic PML media with frequency dependence, given by (8), is causal in the sense of real-axis Fourier inversion contour and, from the discussion in Section II, no dynamic instability should be expected.

\section{B. Complex-Space Formulation: Analytical Properties of the Field Solutions}

For the complex-space PML formulation, the most direct route to investigate the dynamic stability of the Cartesian PML is to simply write down the frequency-domain closedform field solutions (or the Green's functions) for the modified ME's inside the PML and study its analytical behavior in the complex $\omega$-plane. This is because the solutions of the modified ME's inside the PML [3] are just the analytic continuation to complex space of the closed-form solutions of the usual ME's [16], [17], [20]-[22]. Under this analytic continuation, propagating eigenfuctions of ME's obeying Sommerfeld's radiation condition are mapped into exponentially decaying functions, allowing for the reflectionless absorption of EM waves. We shall illustrate this with a simple example.

We take the 2-D Green's function for a cavity enclosed by perfect electric conducting (PEC) walls at $x= \pm d_{x}, y= \pm d_{y}$ (which can be thought of as the FDTD computational domain when using hard boundary conditions), which is written as

$$
\begin{array}{r}
G\left(x, y ; x^{\prime}, y^{\prime}\right) \\
=\sum_{m=0}^{+\infty} \sum_{n=0}^{+\infty} \frac{Z_{m x}\left(x^{\prime}\right) Z_{n y}\left(y^{\prime}\right) Z_{m x}(x) Z_{n y}(y)}{\omega^{2} \mu \epsilon-\left(\frac{m \pi}{2 d_{x}}\right)^{2}-\left(\frac{n \pi}{2 d_{y}}\right)^{2}}
\end{array}
$$

for $-d_{x}<x<d_{x},-d_{y}<y<d_{y}$, where

$$
Z_{p \zeta}(\zeta)=\frac{1}{\sqrt{d_{\zeta}}} \sin \left[k_{p \zeta}\left(\zeta+d_{\zeta}\right)\right]
$$

$k_{p \zeta}=p \pi /\left(2 d_{\zeta}\right), p=m, n, \zeta=x, y$. By inserting a Cartesian PML before the box walls, $G\left(x, y ; x^{\prime}, y^{\prime}\right)$ will be analytically continued to the complex plane through the following mapping on the coordinates (see [16], [17], [21]):

$$
\begin{aligned}
\zeta \rightarrow \tilde{\zeta} & =\zeta_{0}+\int_{\zeta_{0}}^{\zeta} s_{\zeta}\left(\zeta^{\prime \prime}\right) d \zeta^{\prime} \\
& =\zeta_{0}+\int_{\zeta_{0}}^{\zeta}\left(a_{\zeta}+i \frac{\sigma_{\zeta}}{\omega}\right) \\
& =b_{\zeta}(\zeta)+i \frac{\Delta_{\zeta}(\zeta)}{\omega}
\end{aligned}
$$

where $\zeta_{0}$ is on the physical (real) space and $a_{\zeta}=1, \sigma_{\zeta}=0$ in the physical space. From (20), we see that the boundaries of the box are complex valued inside a PML layer $d_{x} \rightarrow \tilde{d}_{x}, d_{y} \rightarrow \tilde{d}_{y}$ and, because of this, the reflection coefficient can be made very small [16], [17]. The function in (18) has an infinite number of poles located along the real $\omega$-axis (natural frequencies of the cavity). With the mapping (20), these poles are translated to the roots of the equation

$$
\omega^{2} \mu \epsilon=\left(\frac{m \pi / 2}{b_{x}\left(d_{x}\right)+i \frac{\Delta_{x}\left(d_{x}\right)}{\omega}}\right)^{2}+\left(\frac{n \pi / 2}{b_{y}\left(d_{y}\right)+i \frac{\Delta_{y}\left(d_{y}\right)}{\omega}}\right)^{2}
$$

which are always located on the real axis or lower half-plane for $a_{\zeta} \geq 1$ and $\sigma_{\zeta} \geq 0$.

In addition to this, it should be noted that $\sin z$ is an entire function (no poles) and that the branch points induced by the $1 / \sqrt{d_{\zeta}}$ factors in (19) (which give rise to simple poles in (21) for this 2-D case) are also on the lower half-plane. Consequently, the resultant PML Green's function is also analytic over the entire upper half $\omega$-plane and causality in the sense of real-axis Fourier inversion contour is preserved. This is true for any observation point $(x, y)$ inside the physical or PML domains. A similar situation occurs in the 3-D case. Consequently, and similarly to the anisotropic-medium PML, the complex-space PML in Cartesian coordinates is dynamically stable.

\section{CYLINDRICAL PML ANALYSIS}

In this section, we examine the causality and dynamic stability of the cylindrical PML. For brevity, the discussion will focus only on those aspects differing from the previous analysis of the Cartesian case.

\section{A. Analytical Properties of the Anisotropic Medium and Complex-Space Formulations}

The cylindrical PML is considered in [16]-[22]. In [20], [21] it is derived for the 2-D case through an analytical continuation (complex stretching) on the radial $\rho$ coordinate. Through this analytic continuation, the propagating eigenfunctions of ME's in cylindrical coordinates obeying Sommerfeld's radiation condition (Hankel functions), which originally have only an algebraic decay on $\rho$, are transformed into functions with exponential decay. In [22], the equations for complex-space 3 -D case are presented, where a simultaneous stretching on the $\rho$ and $z$ coordinates is effected. The anisotropic cylindrical PML 3-D formulation is also presented in [22] (first derived graphically in 2-D in [18]), along with the pertinent fieldmapping equations that relate the complex-space PML and anisotropic PML fields.

In cylindrical coordinates, the constitutive tensor $\overline{\boldsymbol{A}}(\rho, z ; \omega)$ for the anisotropic PML formulation is given by [22]

$$
\bar{\Lambda}(\rho, z ; \omega)=\hat{\rho} \hat{\rho}\left(\frac{s_{\phi} s_{z}}{s_{\rho}}\right)+\hat{\phi} \hat{\phi}\left(\frac{s_{z} s_{\rho}}{s_{\phi}}\right)+\hat{z} \hat{z}\left(\frac{s_{\rho} s_{\phi}}{s_{z}}\right) .
$$

In the above, $s_{\rho}$ and $s_{z}$ are the stretching parameters in the $\rho$ - and $z$-directions respectively, in direct analogy with the Cartesian case

$$
\begin{aligned}
& s_{\rho}(\rho, \omega)=a_{\rho}(\rho)+i \frac{\sigma_{\rho}(\rho)}{\omega} \\
& s_{z}(z, \omega)=a_{z}(z)+i \frac{\sigma_{z}(z)}{\omega}
\end{aligned}
$$


with $a_{\rho, z} \geq 1$ and $\sigma_{\rho, z} \geq 0$. The variable $s_{\phi}$ is a "pseudostretching" parameter in the $\phi$ coordinate that accounts for the modification in the metric coefficient after the stretching in the $\rho$-direction

$$
\begin{aligned}
s_{\phi}(\rho, \omega) & =\frac{\tilde{\rho}}{\rho} \\
& =\frac{1}{\rho}\left(\rho_{0}+\int_{\rho_{0}}^{\rho} s_{\rho}\left(\rho^{\prime}\right) d \rho^{\prime}\right) \\
& =\frac{1}{\rho}\left(b_{\rho}(\rho)+i \frac{\Delta_{\rho}(\rho)}{\omega}\right)
\end{aligned}
$$

where $\rho_{0}$ is on the physical (real) space and $a_{\rho}=1, \sigma_{\rho}=0$ in the physical space. The tensor in (22) can also be written as the product of three simpler $3 \times 3$ uniaxial tensors

$$
\bar{\Lambda}(\rho, z ; \omega)=\bar{\Lambda}_{\rho}(\rho, \omega) \cdot \bar{\Lambda}_{\phi}(\rho, \omega) \cdot \bar{\Lambda}_{z}(z, \omega)
$$

with

$$
\bar{\Lambda}_{\rho}=\hat{\rho} \hat{\rho}\left(\frac{1}{s_{\rho}}\right)+(\hat{\phi} \hat{\phi}+\hat{z} \hat{z}) s_{\rho}
$$

and analogously for $\overline{\boldsymbol{\Lambda}}_{\phi}(\rho, \omega)$ and $\bar{\Lambda}_{z}(z, \omega)$.

In order for $\bar{\Lambda}(\rho, z ; \omega)$ to satisfy causality, each of the tensors in (26) must satisfy it individually. Since the tensors $\overline{\boldsymbol{A}}_{\rho}$ and $\overline{\boldsymbol{A}}_{z}$ have the same analytical properties of the Cartesian PML tensors studied before, we shall limit ourselves to study the analytical properties of the $\overline{\boldsymbol{\Lambda}}_{\phi}(\rho, \omega)$ tensor. The frequency dependence of $\overline{\boldsymbol{\Lambda}}_{\phi}(\rho, \omega)$ (and $\overline{\boldsymbol{\Lambda}}_{\phi}(\rho, \omega)^{-1}$ ) is determined by $s_{\phi}$ and $1 / s_{\phi}$, and, therefore, we must focus our attention on these factors (dependence on the "pseudostretching") and the amount they differ from the Cartesian case. Again, to ensure causality in the sense of a real-axis Fourier inversion contour for $\bar{\Lambda}_{\phi}(\rho, \omega)$, we must ensure (necessary condition) that there are no poles due to $s_{\phi}$ or $1 / s_{\phi}$ above the real axis. For $s_{\phi}$, this is evident, as the only pole is at $\omega=0$, and it can be shown that this function satisfies the Kramers-Kronig relations of the form (11) and (12). However, a major difference arises in the angular factor $1 / s_{\phi}$. It is due to the fact that the factor $\Delta_{\rho}$ in the imaginary part of $s_{\phi}$ may, at certain instances, be negative. This is in contrast to the corresponding $\sigma$ factors in the imaginary part of $s_{x}, s_{y}, s_{z}$, or $s_{\rho}$, which are also chosen to be positive to achieve absorption. Due to the fact that $\sigma_{\rho}$ is positive, when used in (25) for a concave cylindrical PML (at the outer boundary), we will still have $\Delta_{\rho}>0$. However, for a convex cylindrical PML (inner boundary), we have $\Delta_{\rho}<0$, as the integral in (25) is carried over decreasing values of $\rho$. The net effect of this is that the factor $1 / s_{\phi}$ will then have poles in the upper half-plane, and the resultant $\bar{\Lambda}(\rho, z ; \omega)$ will be noncausal in the sense of real-axis Fourier inversion contour. As a consequence (from the discussion in Section II), we should expect dynamic instability on FDTD simulations when employing a convex PML on cylindrical coordinates. Note that we may enforce $\Delta_{\rho}>0$ on convex surfaces if we choose $\sigma_{\rho}<0$ at the convex PML. However, in this case, it would be the factor $1 / s_{\rho}$ which would give rise to singularities in the upper half-plane (this is also related to the fact that, in order to have absorption in the radial direction, $\sigma_{\rho}$ has to be positive, irrespective of the concave or convex geometry).
Such dynamically unstable behavior can also be expected from a direct analysis of the properties of the formal complexspace Green's functions of the cylindrical PML. Analogously to the Cartesian case, the solutions of the modified ME's (frequency domain) inside the cylindrical PML are just the analytic continuation of the solutions of the usual ME's in cylindrical coordinates with the $\rho$ and $z$ coordinates mapped, as in (20), with stretching parameters defined by (23) and (24) [16], [17], [22], so that $\rho \rightarrow \tilde{\rho}$ and $z \rightarrow \tilde{z}$.

For example, the 2-D Green's function for a point source at $\left(\rho^{\prime}, \phi^{\prime}\right)$ inside a PEC cylinder of radius $a$ is given by [23]

$$
\begin{array}{r}
G\left(\rho, \phi ; \rho^{\prime}, \phi^{\prime}\right)=\frac{i}{4} H_{0}^{(1)}\left(k\left|\rho-\rho^{\prime}\right|\right)-\frac{i}{4} \sum_{n=-\infty}^{+\infty} J_{n}(k \rho) \\
\cdot \frac{J_{n}\left(k \rho^{\prime}\right) H_{n}^{(1)}(k a)}{J_{n}(k a) e^{i n\left(\phi-\phi^{\prime}\right)}}
\end{array}
$$

so that, when backed by a concave cylindrical PML, the field maps to

$$
\begin{array}{r}
G\left(\tilde{\rho}, \phi ; \rho^{\prime}, \phi^{\prime}\right)=\frac{i}{4} H_{0}^{(1)}\left(k\left|\tilde{\boldsymbol{\rho}}-\boldsymbol{\rho}^{\prime}\right|\right)-\frac{i}{4} \sum_{n=-\infty}^{+\infty} J_{n}(k \tilde{\rho}) \\
\cdot \frac{J_{n}\left(k \rho^{\prime}\right) H_{n}^{(1)}(k \tilde{a})}{J_{n}(k \tilde{a})} e^{i n\left(\phi-\phi^{\prime}\right)}
\end{array}
$$

where $\tilde{\rho}$ is defined as in (25). Note that $\tilde{a}$ is also complex valued. The effect of the cylindrical PML is to make the reflected field [summation term in (29)] exponentially small [16], [17]. Furthermore, this analytic continuation preserves the analyticity on the upper half-plane of the resultant field in (29). This is because the singularities of (28) are due to branch points (located on the real axis) of the $H_{n}^{(1)}(\cdot)$ and zeros of the denominator functions $J_{n}(\cdot)$ (all on the real axis) [33]. When $\rho \rightarrow \tilde{\rho}$, as in (25), with $\Delta_{\rho}>0$, all singularities are translated down to the lower half $\omega$-plane, so that the upper half-plane is kept free of any singularity for (29).

In contrast, the 2-D Green's function for a point source at $\left(\rho^{\prime}, \phi^{\prime}\right)$ located outside a perfect conducting cylinder of radius $a$ is given by

$$
\begin{gathered}
G\left(\rho, \phi ; \rho^{\prime}, \phi^{\prime}\right)=\frac{i}{4} H_{0}^{(1)}\left(k\left|\rho-\rho^{\prime}\right|\right)-\frac{i}{4} \sum_{n=-\infty}^{+\infty} H_{n}^{(1)}(k \rho) \\
\cdot \frac{H_{n}^{(1)}\left(k \rho^{\prime}\right) J_{n}(k a)}{H_{n}^{(1)}(k a)} e^{-i n\left(\phi-\phi^{\prime}\right)}
\end{gathered}
$$

so that the solution when the perfect conducting cylinder is "coated" by a convex cylindrical PML is given by the analytic continuation

$$
\begin{gathered}
G\left(\tilde{\rho}, \phi ; \rho^{\prime}, \phi^{\prime}\right)=\frac{i}{4} H_{0}^{(1)}\left(k\left|\tilde{\boldsymbol{\rho}}-\boldsymbol{\rho}^{\prime}\right|\right)-\frac{i}{4} \sum_{n=-\infty}^{+\infty} H_{n}^{(1)}(k \tilde{\rho}) \\
\cdot \frac{H_{n}^{(1)}\left(k \rho^{\prime}\right) J_{n}(k \tilde{a})}{H_{n}^{(1)}(k \tilde{a})} e^{-i n\left(\phi-\phi^{\prime}\right)}
\end{gathered}
$$

This analytic continuation does not preserve analyticity in the upper half-plane as the variable $\tilde{\rho}$ now has $\Delta_{\rho}<0$. The zeros of the denominator functions $H_{n}^{(1)}(\cdot)$ located in the lower 


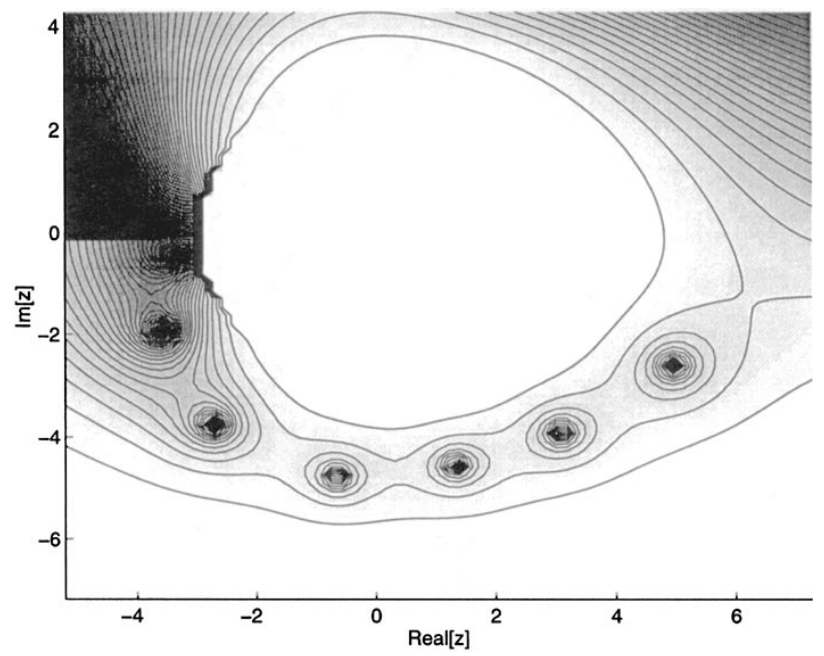

Fig. 1. Contour map of the magnitude of the function $H_{7}^{(1)}(z)$ on the complex plane. Darker regions represent smaller values. The zeros on the lower half-plane and the branch cut on the negative real axis are clearly visible.

half-plane are translated upwards in the complex $\omega$-plane and will eventually appear as poles on the upper half-plane of the reflected field terms in (31). Fig. 1 illustrates the location of the complex zeros of the function $H_{7}^{(1)}(\cdot)$. Branch points of the $H_{n}^{(1)}(\cdot)$ functions on the real axis will also eventually be translated to the upper half-plane. The lack of analyticity on the upper half-plane makes these solutions grow unbounded in the time domain (dynamically unstable behavior), as discussed in Section II.

For generality, we have been focusing on the spectral analysis, but from an analysis of the resulting PML equations in time domain, it is observed that the unstable behavior is usually associated with update equations involving the angular $\phi$ derivatives. This can be seen as a consequence of the way the stretched differential arc lengths behave under stretching for the convex and concave situations. Although the stretched differential arc lengths $d \tilde{z}=s_{z} d z$ and $d \tilde{\rho}=$ $s_{\rho} d \rho$ have positive imaginary parts for both the convex and concave cases, resulting in attenuation for $z$ - and $\rho$ directions in both cases, the stretched angular arc length $\tilde{\rho} d \phi$ has a positive imaginary part for the concave PML and a negative imaginary part for the convex part (the resultant effect, in the concave PML case, is a complex "stretching" on the angular coordinate, but translates, in the convex PML case, to a "squeezing" on the angular coordinate). The negative imaginary part of $\tilde{\rho} d \phi$ gives rise to a temporal operator of the form $(a \partial / \partial t+b)$ with $b / a<0$, associated with equations where the operator $\partial / \partial \phi$ appears. This temporal operator induces exponential growth in time (as opposed to operators with $b / a>0$ obtained with a positive imaginary part of $\tilde{\rho} d \phi$ in the concave case, which induce exponential decay). In connection with this, we note that the singularities in the upper half-plane are solely caused by the angular tensor in (26) $\overline{\boldsymbol{\Lambda}}_{\phi}(\rho, \omega)$. Finally, since the $\phi$-dependence of the fields necessarily obeys a fundamental periodic boundary condition, the fields cannot present a definite growing/attenuative behavior in the $\phi$-direction (in (a)

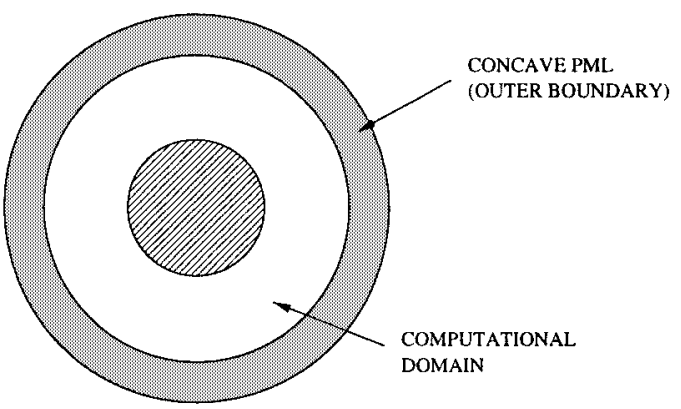

(b)

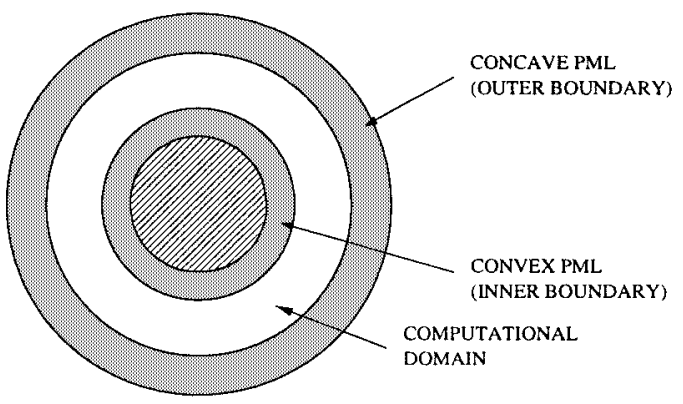

Fig. 2. Configurations for the stability test of the PML-FDTD algorithm in cylindrical and spherical coordinates. The computational domain surrounds a cylindrical or spherical perfectly conducting scatterer (coated or not coated by a covex PML). (a) Configuration A. (b) Configuration B.

other words, the angular spectrum must be real and discrete). Instead, the exponential growing behavior should be present in the time dependence.

\section{B. Numerical Results for the Cylindrical PML}

To illustrate the previous discussion, numerical results of cylindrical grid FDTD simulations with convex and concave cylindrical PML are presented, corresponding to the configurations of Fig. 2(a) and (b).

Fig. 3 shows the normalized $E_{z}$ field from a line source radiating in the presence of a perfectly conducting circular cylinder and computed using a cylindrical grid 2-D PML-FDTD algorithm. The time-stepping scheme is the same as the one described in [20] (complex-space formulation). The space discretization follows the usual staggered-grid centraldifferencing scheme in cylindrical coordinates [34]. The line source is located at $(r, \phi)=\left(4.25 \lambda_{c}, 0^{0}\right)$ and the field is sampled at $(r, \phi)=\left(3.75 \lambda_{c}, 0^{0}\right)$, where $\lambda_{c}$ is the (free space) wavelength corresponding to the central frequency of the excitation pulse. The conducting cylinder is centered at the origin $\rho=0$ and has a radius $a=\lambda_{c}$. The excitation pulse is the first derivative of the Blackman-Harris pulse with central frequency $f_{c}=300 \mathrm{MHz}$. The time step used is $d t=28.6 \mathrm{ps}$.

The cylindrical grid is terminated at $r=5 \lambda_{c}$, where a hard boundary condition is set $\left(E_{z}=0\right)$. In all simulations, the FDTD algorithm includes an eight-layer concave cylindrical PML region before the grid termination (at the outer boundary). The PML thickness is $0.5 \lambda_{c}$ for a cell size $\Delta \rho=\lambda_{c} / 16$ in the radial direction. A quadratic taper on $\sigma_{\rho}$ is used, varying from zero up to $\sigma_{\rho, \max }$. For simplicity, $a_{\rho}=1$ everywhere. The solid line in Fig. 2 shows the result when using only 


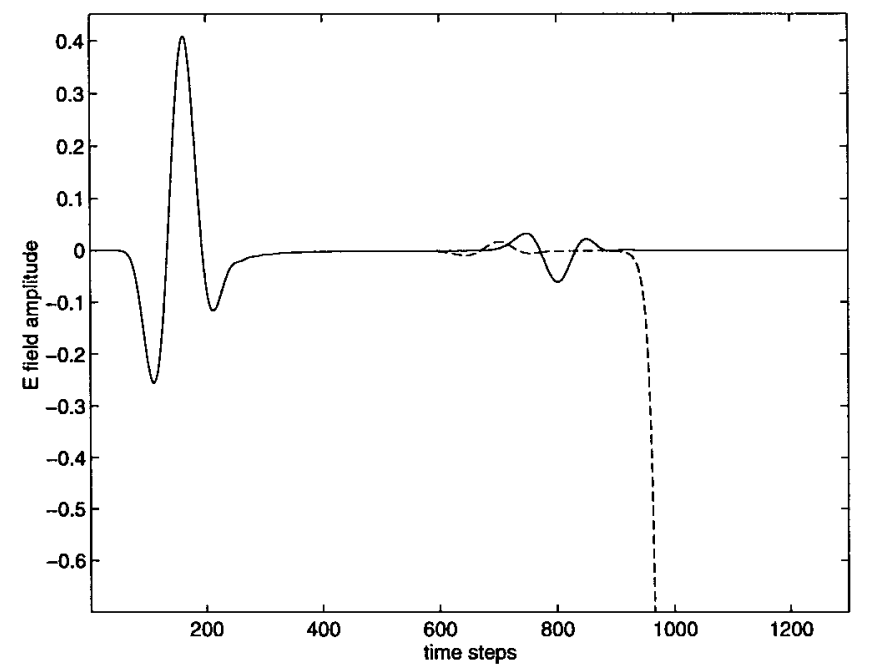

Fig. 3. 2-D cylindrical-grid FDTD solution with eight-layer cylindrical PML. The solid line represents the case with the concave cylindrical PML only. Both the direct and reflected pulse due to the inner PEC cylinder are visible. No reflected fields due to the grid ends at the outer boundary are visible. The dashed line represents the FDTD simulation with the convex cylindrical PML coating the inner PEC cylinder. The dramatic early-time instability incurred is evident.

a concave cylindrical PML before the outer boundary. This corresponds to the configuration depicted in Fig. 2(a). Both the direct pulse and nonspurious reflected pulse due to the inner perfect conducting cylinder are visible. No spurious reflection due to the outer boundary is visible. The dotted line is the result of the simulation when an eight-layer convex cylindrical PML is placed around the inner cylinder. This corresponds to the configuration depicted in Fig. 2(b). The same value of $\sigma_{\rho, \max }$ is used for the concave and convex PML's in this case. A reflected wave from the (PML coated) inner cylinder is nonetheless present and, more importantly, the instability of the resultant FDTD algorithm in this case is dramatic. It occurs soon after the wave reaches the convex PML (early-time effect).

Fig. 4 shows the results of the same simulation, but also with smaller values for $\sigma_{\rho, \max }$ in the convex cylindrical PML. This illustrates that the growth rate of the electric field inside the convex cylindrical PML is proportional to $\sigma_{\rho \text {, } \max }$, as expected.

\section{Imposing Stability a Posteriori: The Quasi-PML}

The conclusions above have impact on the design of EM PML absorbers both as tools for numerical simulations and on their use (anisotropic-medium formulation) as a physical basis for engineered artificial absorbers [35].

An alternative to avoid the singularities in the upper halfplane for the convex case would be to impose $\Delta_{\rho} \geq 0$ in (25) for the inner boundary (irrespective of $\sigma_{\rho} \geq 0$ ). In this manner, a dynamically stable scheme can be obtained. However, the resultant cylindrical interface is not perfectly matched anymore (since (25) is not true anymore). It should, more appropriately, be called a quasi-PML [36]. This approximation behaves as a true PML only in the limit $\rho \rightarrow \infty$, when the cylindrical PML reduces to the Cartesian PML. From our preliminary

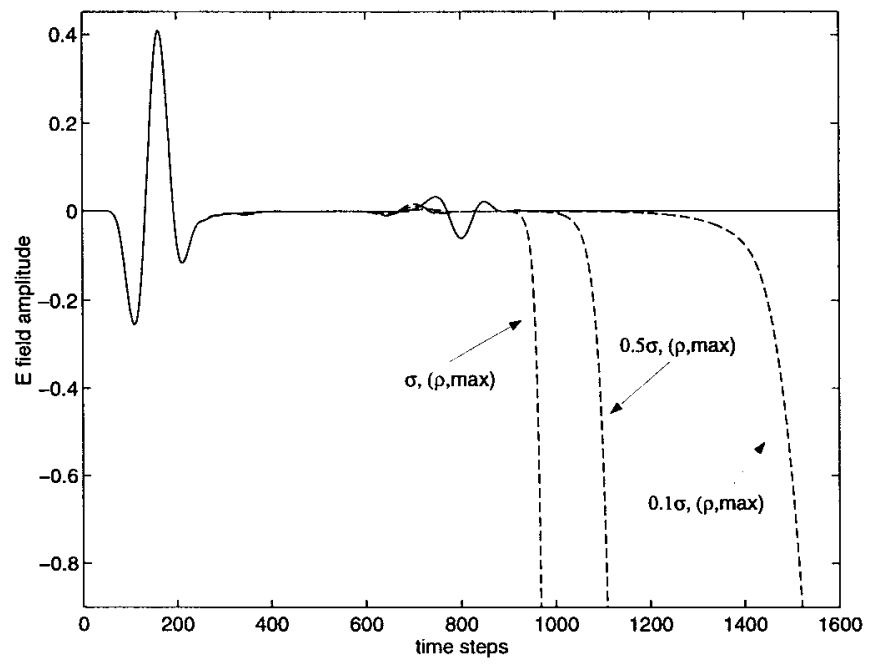

Fig. 4. Same simulation as the previous figure, but using different values for $\sigma_{\rho, \max }$ in the convex PML. The growth rate of the fields gets smaller as the value of $\sigma_{\rho, \max }$ is decreased (smaller stretching).

experience, the two important features exhibited by a quasiPML when compared to a true PML (when the latter is applicable) are: 1) a nonzero reflection coefficient in the continuum limit and 2) the need for a more finely tuned profile to achieve the best results.

Still, a quasi-PML may have practical applications when $\rho \gg \lambda$, for both numerical simulations and as a physical basis for engineered artificial absorbers.

\section{SPHERICAL PML ANALYSIS}

In this section, we examine the causality and dynamic stability of the spherical PML. The focus is on those aspects that differ from the previous cases.

\section{A. Analytical Properties of the Anisotropic Medium and Complex-Space Formulations}

The spherical PML is considered in [16], [17], [20], and [22]. In [20], it is derived through an analytical continuation (complex stretching) on the radial $r$ coordinate. The anisotropic spherical PML formulation is presented in [22], along with the pertinent field-mapping equations that relate the complex-space PML and anisotropic PML fields.

In the spherical system, the constitutive tensor $\bar{\Lambda}(\rho, z ; \omega)$ for the anisotropic PML formulation is given by [22]

$$
\bar{\Lambda}(r ; \omega)=\hat{r} \hat{r}\left(\frac{s_{\theta} s_{\phi}}{s_{r}}\right)+\hat{\theta} \hat{\theta}\left(\frac{s_{\phi} s_{r}}{s_{\theta}}\right)+\hat{\phi} \hat{\phi}\left(\frac{s_{r} s_{\theta}}{s_{\phi}}\right) .
$$

In the above, $s_{r}$ is the stretching parameter in the $r$-direction, in direct analogy with the Cartesian case

$$
s_{r}(r, \omega)=a_{r}(r)+i \frac{\sigma_{r}(r)}{\omega}
$$

with $a_{r} \geq 1$ and $\sigma_{r} \geq 0$. The variables $s_{\theta}$ and $s_{\phi}$ are "pseudostretching" parameters in the $\theta$ and $\phi$ angular coordinates that account for the modification in the metric coefficient after 
the stretching in the $r$-direction

$$
\begin{aligned}
s_{\theta}(r, \omega) & =s_{\phi}(r, \omega) \\
& =\frac{\tilde{r}}{r} \\
& =\frac{1}{r}\left(r_{0}+\int_{r_{0}}^{r} s_{r}\left(r^{\prime}\right) d r^{\prime}\right) \\
& =\frac{1}{r}\left(b_{r}(r)+i \frac{\Delta_{r}(r)}{\omega}\right)
\end{aligned}
$$

where $r_{0}$ is on the physical (real) space and $a_{r}=1, \sigma_{r}=0$ in the physical space.

Since $s_{\theta}=s_{\phi}$, the constitutive tensor in (32) can be simplified to

$$
\bar{\Lambda}(r ; \omega)=\hat{r} \hat{r}\left(\frac{\tilde{r}}{r}\right)^{2}\left(\frac{1}{s_{r}}\right)+(\bar{I}-\hat{r} \hat{r}) s_{r} .
$$

Following the same reasoning used in the Cartesian PML and cylindrical PML cases, it can be shown that $\overline{\boldsymbol{\Lambda}}(r ; \omega)$ has no poles in the upper half-plane for a concave spherical PML and, therefore, the resultant FDTD scheme will be dynamically stable in this case.

In the case of a convex spherical PML, we note that, due to the pole cancellation in the angular terms $\hat{\theta} \hat{\theta}$ and $\hat{\phi} \hat{\phi}$ in (32), the tensor $\bar{\Lambda}(r ; \omega)$ has no poles in the upper half-plane. However, its inverse $\bar{\Lambda}(r ; \omega)^{-1}$ has poles in the upper halfplane due to the factor $\tilde{r} / r$ in the radial term $\hat{r} \hat{r}$ of (35), and, therefore, dynamical instability should be expected on FDTD simulation using a convex PML on spherical coordinates.

This is also predicted by a direct analysis of the field solutions in the frequency domain. Analogously to the Cartesian and cylindrical cases, the solutions of the modified ME's inside the spherical PML are just the analytic continuation of the solutions of the usual ME's in spherical coordinates, with the $r$ coordinate mapped as in (20) with stretching parameters defined by (33) [16], [17], [20], [22], so that $r \rightarrow \tilde{r}$. When using a convex spherical PML, the analyticity of the solutions in the upper half-plane is again not preserved. The important distinction from the cylindrical case discussed in Section IV is that the Hankel $H_{n}^{(1)}(\cdot)$ and Bessel $J_{n}(\cdot)$ functions are replaced by its spherical counterparts $h_{n}^{(1)}(\cdot)$ and $j_{n}(\cdot)$. Although not having branch points on the real axis as $H_{n}^{(1)}(\cdot)$, the denominator functions $h_{n}^{(1)}(\cdot)$ in the spherical case still have zeros on the lower half-plane, as illustrated in Fig. 5. These zeros will eventually appear as poles on the upper half-plane of the field solutions under complex stretching.

The same observations made about the quasi-PML in cylindrical coordinates also applies to the spherical case. The inner/outer domain differentiation is a major asymmetry present on the cylindrical or spherical PML and has no analogy in the Cartesian PML. The spatial domain enclosed by an inner boundary on the cylindrical or spherical is of finite extent, in contrast to the Cartesian and outer boundaries cases. A wave propagating into an inner cylindrical or spherical domain eventually emerges back on the outer domain, but the converse is not true. Moreover, a strictly concave or planar PML cannot completely coat a body of finite size.

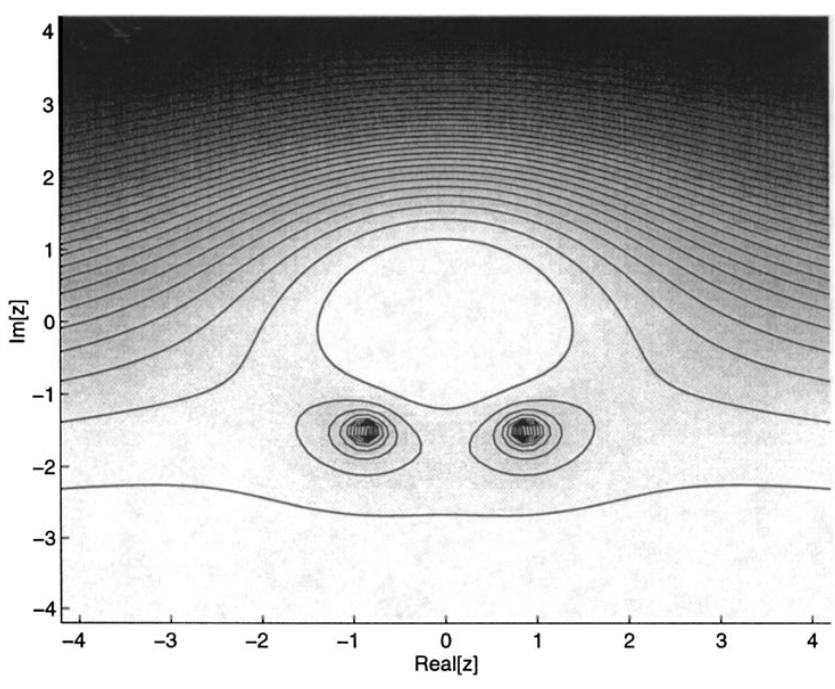

Fig. 5. Contour map of the magnitude of the function $h_{2}^{(1)}(z)$ on the complex plane. Darker regions represent smaller values. The zeros on the lower half-plane are clearly visible.

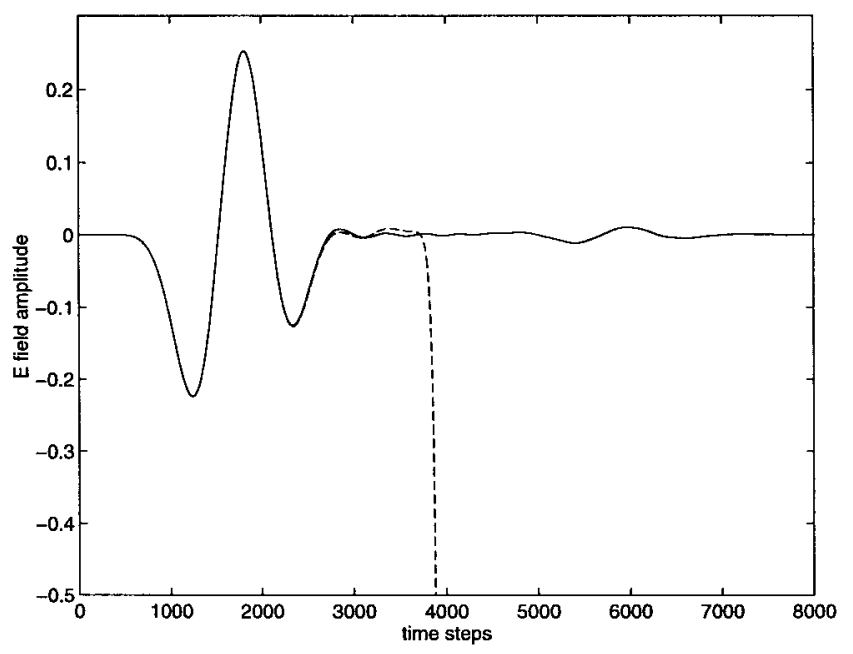

Fig. 6. 3-D spherical-grid FDTD solution with eight-layer spherical PML. The solid line represents the case with the concave spherical PML only. Both the direct and reflected pulse due to the inner PEC sphere are visible. No reflected fields due to the grid ends at the outer boundary are visible. The dashed line represents the FDTD simulation with the convex spherical PML coating the inner PEC sphere. The dramatic early-time instability incurred is evident.

\section{B. Numerical Results for the Spherical PML}

In what follows, some numerical results for FDTD simulations using the spherical PML are presented to illustrate the previous discussion.

Fig. 6 depicts the normalized $E_{\theta}$ field from a Hertzian dipole radiating in the presence of a perfect conducting sphere. The field is computed using a spherical-grid 3-D PML-FDTD algorithm. The time-stepping scheme is the same as the one described in [20] (complex-space formulation). The space discretization and the treatment of the singularities of the spherical coordinate system follows [37]. The $\theta$-polarized Hertzian dipole is located at $(r, \theta, \phi)=\left(1.9 \lambda_{c}, 90^{\circ}, 0^{\circ}\right)$ and the resultant field is sampled at $(r, \theta, \phi)=\left(2.5 \lambda_{c}, 90^{\circ}, 0^{0}\right)$, where $\lambda_{c}$ is the (free-space) wavelength corresponding to 


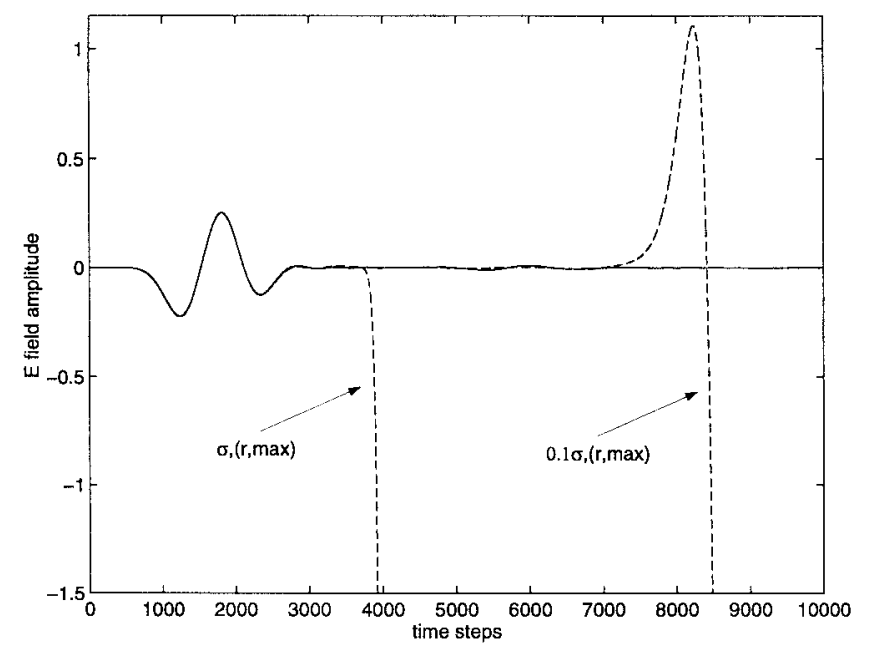

Fig. 7. Same simulation as the previous figure with another simulation using a smaller value for $\sigma_{r, \max }$ in the convex PML. Similarly to the cylindrical case, the growth rate of the fields becomes smaller as the complex stretching is decreased.

the central frequency of the excitation pulse. The perfectly conducting sphere is centered at the origin $r=0$ and has a radius $a=0.5 \lambda_{c}$. The spherical grid is terminated at $r=3.5 \lambda_{c}$, where a hard boundary condition is set (zero tangential fields). The FDTD algorithm includes an eight-layer concave spherical PML region before the grid ends. The PML thickness is $0.8 \lambda_{c}$ for a cell size $\Delta r=\lambda_{c} / 10$ in the radial direction. A quadratic taper on $\sigma_{r}$ is used, varying from zero to $\sigma_{r, \max }$. For simplicity, $a_{r}$ is set equal to one everywhere. The excitation pulse is the first derivative of the Blackman-Harris pulse with central frequency $f_{c}=300 \mathrm{MHz}$. Due to the high grid curvature on the simulation region, a very small time step has to be used: $d t=2.45$ ps. The solid line in the Fig. 6 shows the result when using only a concave spherical PML before the outward boundary. This corresponds to the configuration depicted in Fig. 2(a). Both the direct pulse and nonspurious reflected pulse due to the inner perfect conducting sphere are visible. No spurious reflection due to the outer boundary is visible. The small oscillations after the passage of the direct pulse are due to the numerical dispersion effects caused by the coarse grid density adopted. The dotted line is the result of the simulation when an eight-layer convex spherical PML is placed around the inner sphere. This corresponds to the configuration depicted in Fig. 2(b). The same value of $\sigma_{r, \max }$ is used for the concave and convex PML in this case. As in the cylindrical case, we observe the dramatic instability of the resultant FDTD algorithm, with an exponential growth soon after the wave reaches the convex spherical PML.

Fig. 7 shows the results of the same simulation using also a smaller value for $\sigma_{r, \max }$ in the convex spherical PML. The instability is still present, but appears later.

\section{Relation Between Anisotropic Medium PML AND COMPLEX-SPACE PML FIELDS}

Although, for clarity, we have addressed the anisotropicmedium and complex-space formulations for the PML separately here, their dynamic stability properties are indeed intimately connected. The results obtained also seem to suggest that. Such connection is best appreciated by writing down explicitly, for the three coordinate systems considered, the formulas relating the resulting fields inside the PML in the two formulations [22]. They have the very simple generic form

$$
E_{\zeta}^{a}(\boldsymbol{r} ; \omega)=s_{\zeta}(\boldsymbol{r} ; \omega) E_{\zeta}^{c}(\boldsymbol{r} ; \omega)
$$

where $\zeta$ stands for the coordinates, $\zeta=x, y, z, \rho, r, \phi, \theta$, and $s_{\zeta}$ stands for the stretching or pseudostretching variables in the appropriate directions, as defined before. The superscripts " $a$ " and " $c$ " in (36) stand for anisotropic PML and complexspace PML fields, respectively. If we interpret the complex stretching as a stretching on the metric of space [38], then we conclude that the stretching or pseudostretching $s_{\zeta}$ variables in (36) are just the factors that multiply the original metric coefficients to give the new (stretched) metric coefficients on each direction. This interpretation, in view of the metric invariance of ME's [38], [39], also explains the reason why there exist two different PML formulations. As discussed before, from the knowledge of the closed-form solutions in free-space $E_{\zeta}(\boldsymbol{r} ; \omega)$, we may directly write down the closedform field solutions inside the complex-space PML $E_{\zeta}^{c}(\boldsymbol{r} ; \omega)$ through a simple analytic continuation on the spatial variables of $E_{\zeta}(\boldsymbol{r} ; \omega)$ given by (20), (25), or (34). If we denote such analytic continuation more compactly as $\boldsymbol{r} \rightarrow \tilde{\boldsymbol{r}}$, then we have

$$
E_{\zeta}^{c}(\boldsymbol{r} ; \omega)=E_{\zeta}(\tilde{\boldsymbol{r}} ; \omega)
$$

By combining (36) and (37), we may also write the field solutions inside the anisotropic PML in closed-form in terms of the free-space solutions

$$
E_{\zeta}^{a}(\boldsymbol{r} ; \omega)=s_{\zeta}(\boldsymbol{r} ; \omega) E_{\zeta}(\tilde{\boldsymbol{r}} ; \omega)
$$

Multiplying (36) by $i \omega$ and transforming back to the time domain, we have

$$
\frac{\partial}{\partial t} E_{\zeta}^{a}(t)=\left(\alpha \frac{\partial}{\partial t}+\beta\right) E_{\zeta}^{c}(t)
$$

where $\alpha$ and $\beta$ are functions depending on the stretching parameters. In the physical domain, $\alpha=1, \beta=0$, and the fields coincide.

\section{CONCLUSIONS}

The analytic continuation (complex coordinate stretching) of ME's to complex space is a powerful method to achieve the reflectionless absorption of EM waves in different coordinate systems. Through this approach, it is possible to extend the PML concept to different coordinate systems and even to other classes of linear-wave phenomena, such as scalar (Helmholtz equation) [40], paraxial [15], and elastic wave problems [14].

In this paper, we have pointed out some limitations of this approach. In particular, the complex stretching should preserve analyticity on the upper half of the complex $\omega$-plane to ensure that causality is not violated.

We have addressed the connection between the violation of causality in the sense of a real-axis Fourier inversion contour and the dynamic instability of the resultant time-stepping scheme in the FDTD method. 
We have shown that the original Cartesian anisotropic PML does indeed satisfy the Kramers-Kronig relations and, therefore, does not violate causality in the sense of real-axis Fourier inversion contour. In addition, the resultant fields on the complex-space formulation preserve the analyticity on the upper half-plane and, as a result, the Cartesian PML is dynamically stable.

In the cylindrical and spherical case, the anisotropic-medium formulation of the concave PML has constitutive tensors free of singularities and zeros on the upper half-plane. For the convex anisotropic PML (i.e., at inner boundaries), however, the resultant constitutive tensors contain singularities and/or poles on the upper half-plane. For the complex-space formulation of the concave PML (i.e., at outer boundaries), both in cylindrical and spherical coordinates, analyticity of the frequency-domain field solutions on the upper half-plane is preserved. In the convex case, however, the field solutions contain singularities in the upper half-plane. These solutions are noncausal in the sense of a real-axis Fourier inversion contour. The lack of analyticity on the upper half-plane implies a dynamically unstable FDTD scheme with solutions that may grow unbounded. This is illustrated with numerical results from 2-D cylindrical grid and 3-D spherical grid FDTD simulations.

We have also pointed out that the anisotropic PML fields and complex-space PML fields are related through a set of simple equations. Furthermore, from the knowledge of the closedform free-space solutions, both fields can be written in closed form.

To avoid dynamical instability in the convex case, a quasiPML might be an alternative for large radius of curvature. In principle, the analysis presented here does not completely rule out the case for a convex PML on cylindrical and spherical surfaces. Instead, it points out that the same method used to derive a concave PML cannot be naively applied to obtain a convex PML. The conclusions obtained are based on the particular frequency dependence chosen for the complex stretching variables $s_{\zeta}(\omega)$, which is the one prevalent in the literature. However, other choices are also possible to obtain different functional dependences on $\omega$ (at the cost of increased complexity on the resultant time-domain equations). It is of interest to investigate whether, by doing so, it might be possible to derive a stable PML on convex surfaces. Finally, the analysis presented here provides the basis for a local analysis of the analytical properties and dynamical stability of the conformal PML on a general orthogonal curvilinear coordinate system [41].

\section{REFERENCES}

[1] J. Berenger, "A perfectly matched layer for the absorption of electromagnetic waves," J. Comput. Phys., vol. 114, pp. 185-200, 1994.

[2] _ "Three-dimensional perfectly matched layer for the absorption of electromagnetic waves," J. Comput. Phys., vol. 127, pp. 363-379, 1996.

[3] W. C. Chew and W. Weedon "A 3-D perfectly matched medium from modified Maxwell's equations with stretched coordinates," Microwave Opt. Technol. Lett., vol. 7, pp. 599-604, 1994.

[4] D. S. Katz, E. T. Thiele, and A. Taflove, "Validation and extension to three dimensions of the Berenger PML absorbing boundary condition," IEEE Microwave Guided Wave Lett., vol. 4, pp. 268-270, Aug. 1994.
[5] C. M. Rappaport, "Interpreting and improving the PML absorbing boundary condition using anisotropic lossy mapping of space," IEEE Trans. Magn., vol. 32, pp. 968-974, Mar. 1996.

[6] Z. S. Sacks, D. M. Kingsland, R. Lee, and J.-F. Lee, "A perfectly matched anisotropic absorber for use as an absorbing boundary condition," IEEE Trans. Antennas Propagat., vol. 43, pp. 1460-1463, Dec. 1995.

[7] S. D. Gedney, "An anisotropic PML absorbing media for the FDTD simulation of fields in lossy and dispersive media," Electromagn., vol. 16, pp. 399-415, 1996.

[8] _ "An anisotropic perfectly matched layer absorbing medium for the truncation of FDTD lattices," IEEE Trans. Antennas Propagat., vol. 44, pp. 1630-1639, Dec. 1996.

[9] L. Zhao and A. C. Cangellaris, "GT-PML: Generalized theory of perfectly matched layers and its application to the reflectionless function of finite-difference time-domain grids," IEEE Trans. Microwave Theory Tech., vol. 44, pp. 2555-2563, Dec. 1996.

[10] W. C. Chew and J. M. Jin, "Perfectly matched layers in the discretized space: an analysis and optimization," Electromagn., vol. 16 pp. 325-340, 1996.

[11] N. Kantartzis and T. Tsiboukis, "A comparative study of the Berenger perfectly matched layer, the superabsorption technique and several highorder ABC's for the FDTD algorithm in two and three dimensional problems," IEEE Trans. Magn., vol. 33, pp. 1460-1463, Mar. 1997.

[12] J. -Y. Wu, D. M. Kingsland, J. -F. Lee, and R. Lee, "A comparison of anisotropic PML to Berenger's PML and its application to the finiteelement method for EM scattering," IEEE Trans. Antennas Propagat., vol. 45, pp. 40-50, Jan. 1997.

[13] M. Kuzuoglu and R. Mittra, "Investigation of nonplanar perfectly matched absorbers for finite-element mesh truncation," IEEE Trans. Antennas Propagat., vol. 45, pp. 474-486, Mar. 1997.

[14] W. C. Chew and Q. H. Liu, "Perfectly matched layers for elastodynamics: a new absorbing boundary condition," J. Comput. Acoust., vol. 4 pp. 341-359, 1996.

[15] F. Collino, "Perfectly matched absorbing layers for the paraxial equations," J. Comput. Phys., vol. 131, pp. 164-180, 1997.

[16] W. C. Chew, J. M. Jin, and E. Michielssen, "Complex coordinate system as a generalized absorbing boundary condition," in Proc. 13th Annu. Rev. Prog. Appl. Comput. Electromag., vol. 2, Monterey, CA, Mar. 17-21, 1997, pp. 909-914.

[17] _ "Complex coordinate stretching as a generalized absorbing boundary condition," Microwave Opt. Technol. Lett., vol. 15, pp. 363-369, 1997

[18] J. Maloney, M. Kesler, and G. Smith, "Generalization of PML to cylindrical geometries," in Proc. 13th Annu. Rev. Prog. Appl. Comput. Electromag., vol. 2, Monterey, CA, Mar. 17-21, 1997, pp. 900-908.

[19] B. Yang, D. Gottlieb, and J. S. Hesthaven, "On the use of PML ABC's in spectral time-domain simulations of electromagnetic scattering," in Proc. 13th Аnnu. Rev. Prog. Appl. Comput. Electromag., vol. 2, Monterey, CA, Mar. 17-21, 1997, pp. 926-933.

[20] F. L. Teixeira and W. C. Chew, "PML-FDTD in cylindrical and spherical grids," IEEE Microwave Guided Wave Lett., vol. 7, pp. 285-287, Sept. 1997.

[21] F. Collino and P. Monk, "The perfectly matched layer in curvilinear coordinates," SIAM J. Sci. Stat. Comput., vol. 19, pp. 2061-2090, 1998.

[22] F. L. Teixeira and W. C. Chew, "Systematic derivation of anisotropic PML absorbing media in cylindrical and spherical coordinates," IEEE Microwave Guided Wave Lett., vol. 7, pp. 371-373, Nov. 1997.

[23] W. C. Chew, Waves and Fields in Inhomogeneous Media. New York: Van Nostrand, 1990.

[24] E. M. Lifshitz and L. P. Pitaevskii, Statistical Physics: Part 1. New York: Pergamon, 1980, pp. 377-384.

[25] L. D. Landau, E. M. Lifshitz, and L. P. Pitaevskii, Electrodynamics of Continuous Media. New York: Pergamon, 1984, pp. 279-283.

[26] H. M. Nussenzveig, Causality and Dispersion Relations. New York: Academic, 1972, pp. 43-47.

[27] J. W. Nehrbass, J. -F. Lee, and R. Lee, "Stability analysis for perfectly matched layered absorbers,"Electromagn., vol. 16, pp. 385-397, 1996.

[28] S. Abarbanel and D. Gottlieb, "A mathematical analysis of the PML method," J. Comput. Phys., vol. 134, pp. 357-363, 1997.

[29] J. A. Kong, Electromagnetic Wave Theory. New York: Wiley, 1982, pp. 297-299, 344-345.

[30] P. C. Martin, "Sum rules, Kramers-Kronig relations, and transport coefficients in charged systems," Phys. Rev., vol. 161, pp. 143-155, 1967.

[31] M. Kuzuoglu and R. Mittra, "Frequency dependence of the constitutive parameters of causal perfectly matched anisotropic absorbers," IEEE Microwave Guided Wave Lett., vol. 6, pp. 447-449, Dec. 1996. 
[32] J. Hilgervoord, Dispersion Relations and Causal Description. Amsterdam, The Netherlands: North-Holland, 1960, pp. 40-49.

[33] M. Abramowitz and I. A. Stegun, Handbook of Mathematical Functions. New York: Dover, 1970, pp. 372, 373, 441.

[34] M. Fusco, "FDTD algorithm in curvilinear coordinates," IEEE Trans. Antennas Propagat., vol. 38, pp. 76-89, Jan. 1990.

[35] R. W. Ziolkowsky, "The design of Maxwellian absorbers for numerical boundary conditions and for practical applications using engineered artificial materials," IEEE Trans. Antennas Propagat., vol. 45, pp. 656-671, Apr. 1997.

[36] J. Q. He and Q. H. Liu, "A nonuniform cylindrical FDTD algorithm with improved PML and quasi-PML absorbing boundary conditions," IEEE Trans. Geosci. Remote Sensing, vol. 37, pp. 1066-1072, Mar. 1999.

[37] R. Holland, "THREDS: A finite-difference time-domain EMP code in 3D spherical coordinates,"IEEE Trans. Nucl. Sci., vol. 30, pp. 4592-4595, Dec. 1983.

[38] F. L. Teixeira and W. C. Chew, "Differential forms, metrics, and the reflectionless absorption of electromagnetic waves," J. Electromagn. Wave Applicat., vol. 13, pp. 665-686, 1999.

[39] "Lattice electromagnetic theory from a topological viewpoint," J. Math. Phys., vol. 40, no. 1, pp. 169-187, 1999.

[40] "Extension of the PML absorbing boundary condition to 3-D spherical coordinates: Scalar case," IEEE Trans. Magn., vol. 34, pp. 2680-2683, Sept. 1998.

[41] _ "Analytical derivation of a conformal perfectly matched absorber for electromagnetic waves," Microwave Opt. Technol. Lett., vol. 17, pp. 231-236, 1998

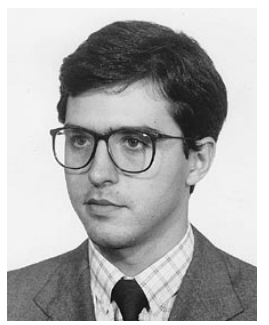

F. L. Teixeira received the B.S. and M.S. degrees in electrical engineering from the Pontifical Catholic University of Rio de Janeiro (PUC-Rio), Rio de Janeiro, Brazil, in 1991 and 1995, respectively, and is currently working toward the Ph.D. degree in electrical engineering at the University of Illinois at Urbana-Champaign.

While at PUC-Rio, he conducted research both in the Department of Electrical Engineering and in the Department of Physics. From 1992 to 1994, he was a Technical Officer with the Military Institute of Engineering, Rio de Janeiro, and the Brazilian Army R\&D Center (IPDCTEx). From 1994 to 1996, he was a Member of the Technical Staff in the Satellite Transmission Department, EMBRATEL S.A. (Brazilian Telecom), Rio de Janeiro, Brazil. Since 1996, he has been a Research Assistant with the Center for Computational Electromagnetics, University of Illinois at UrbanaChampaign. His current research interests include wave propagation and scattering modeling for communication, sensing, and device applications. His previous research includes integral-equation analysis of reflector antennas, synthesis of shaped beam reflectors, surface interpolation techniques, and simulation of lattice gauge theories. He has co-authored 40 refereed papers in these areas.

Mr. Teixeira is a member of Phi Kappa Phi. He was a recipient of scholarships from the Brazilian agencies FAPERJ, TELEBRAS, and CNPq, a CAPES/Brasilia Fellowship (1996-1999), a 1998-1999 IEEE MTT-S Graduate Fellowship Award, a 1999 USNC/URSI Student Paper Competition Prize, and the Raj Mittra Outstanding Research Award presented by the University of Illinois at Urbana-Champaign.

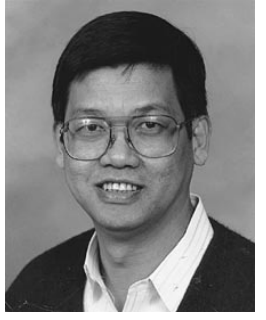

W. C. Chew (S'79-M'80-SM'86-F'93) received the B.S., M.S., and Engineer's degrees, and the $\mathrm{Ph} . \mathrm{D}$. degree from the Massachusetts Institute of Technology (MIT), Cambridge, in 1976, 1978, and 1980, respectively, all in electrical engineering.

From 1981 to 1985, he was with Schlumberger-Doll Research, Ridgefield, CT. While there, he was a Program Leader and later a Department Manager. From 1985 to 1990, he was an Associate Professor with the University of Illinois at UrbanaChampaign, where he currently is a Professor teaching graduate courses in waves and fields in inhomogeneous media, and theory of microwave and optical waveguides and supervising a graduate research program. From 1989 to 1993, he was the Associate Director the Advanced Construction Technology Center, University of Illinois at UrbanaChampaign, and is currently the Director of the Center for Computational Electromagnetics and the Electromagnetics Laboratory. He has authored Waves and Fields in Inhomogeneous Media (Piscataway, NJ: IEEE Press, 1995), published over 200 scientific journal articles, and presented over 230 conference papers. His recent research interest has been in the area of wave propagation, scattering, inverse scattering, and fast algorithms related to scattering, inhomogeneous media for geophysical subsurface sensing, and nondestructive testing applications. He has also previously analyzed electrochemical effects and dielectric properties of composite materials, microwave and optical waveguides, and microstrip antennas. His name is often listed in the List of Excellent Instructors on the University of Illinois at Urbana-Champaign campus. He is an associate editor for the Journal of Electromagnetic Waves and Applications (1996-present), and Microwave Optical Technology Letters (1996-present). He was also an associate editor with the International Journal of Imaging Systems and Technology (1989-1994), and has been a guest editor of Radio Science (1986), the International Journal of Imaging Systems and Technology (1989), and Electromagnetics (1995).

Dr. Chew is a member of Eta Kappa Nu, Tau Beta Pi, URSI Commissions $\mathrm{B}$ and F, and is an active member with the Society of Exploration Geophysics (SEG). He was an National Science Foundation (NSF) Presidential Young Investigator in 1986. He was also an AdCom member of the IEEE Geoscience and Remote Sensing Society. He is currently an associate editor of the IEEE Transactions of Geoscience and Remote SENSING (1984-present). 